\title{
Clinical Versatility of the Functional Lumen Imaging Probe in Pediatric Gastrointestinal Procedures
}

\author{
Jacqueline M Barsamian ${ }^{1}$, Armen Gharibans ${ }^{2,3}$, Alain J Benitez ${ }^{4}$ and Hayat M Mousa ${ }^{1 *}$ \\ ${ }^{1}$ Division of Gastroenterology, Hepatology and Nutrition, Department of Pediatrics, University of California, Rady Children's Hospital, San Diego, USA \\ ${ }^{2}$ Department of Surgery, University of Auckland, New Zealand
}

${ }^{3}$ Auckland Bioengineering Institute, University of Auckland, New Zealand

${ }^{4}$ Department of Gastroenterology, Hepatology and Nutrition, Children's Hospital of Philadelphia; Department of Pediatrics, Perelman School of Medicine, USA

*Corresponding author: Hayat M Mousa, Division of Gastroenterology, Hepatology and Nutrition, Department of Pediatrics, University of California, San Diego, Rady Children's Hospital, San Diego, 3020 Children's Way M.C. 5030, San Diego, CA 92123, USA.

To Cite This Article: Jacqueline MB, Armen G, Alain JB, Hayat MM. Clinical Versatility of the Functional Lumen Imaging Probe in Pediatric Gastrointestinal Procedures. 2020 - 9(4). AJBSR.MS.ID.001413. DOI: 10.34297/AJBSR.2020.09.001413.

Received: 眥 July 08, 2020; Published: 鲇 July 13, 2020

\begin{abstract}
The functional lumen imaging probe (FLIP) is a novel device with great utility as a diagnostic and therapeutic tool for a variety of gastrointestinal conditions. While its use is well documented within the adult population, very few articles have described the use of the FLIP in the pediatric population. We summarize current literature describing the use of the FLIP in the pediatric population as well as our experiences with the FLIP for the past five years in patients with achalasia, esophageal dysphagia, and/or eosinophilic esophagitis (EoE) at two pediatric centers.

Keywords: Pediatrics; Functional Lumen Imaging Probe, EndoFLIP, EsoFLIP, Eosinophilic Esophagitis, Achalasia, Esophageal Stenosis, EoE, Peroral Endoscopic Myotomy

Abbreviations: CSA: Cross-sectional Area; EoE: Eosinophilic Esophagitis; eos/hpf Eosinophils per High Power Field; EGJ: Esophogastric Junction; FLIP: Functional Lumen Imaging Probe; LHM: Laparoscopic Heller Myotomy; POEM: Peroral Endoscopic Myotomy
\end{abstract}

\section{Introduction}

The functional lumen imaging probe (FLIP) is a novel tool that has been utilized both diagnostically and therapeutically for a variety of conditions in a range of populations. In the setting of gastroenterology, the FLIP may be used to measure the upper esophageal sphincter, esophageal body, lower esophageal sphincter, pylorus, and anal sphincter in a variety of clinical scenarios and settings [1]. Using high-resolution impedance planimetry, the FLIP determines the pressure-geometry relationship or distensibility of hollow gastrointestinal organs including the esophagus. FLIP is currently available in the form of two different iterations: the endoluminal functional lumen imagine probe (EndoFLIP) and the EsoFLIP which is geared towards esophageal treatment. While current common endoscopic tools are indeed visually helpful, they lack in terms of precision and are inept when it comes to accurately deciphering important luminal characteristics such as esophageal distensibility and compliance. The FLIP can therefore safely and accurately provide important luminal characteristics in both the adult and pediatric patient populations.

The utility of the FLIP follows the methods and management strategies for many pediatric conditions in that it has been thus far largely adapted from its use in the adult population. Its utility in pediatric patients, however, has yet to earn the same recognition as few publications detailing its use in pediatrics currently exist. Additionally, the use of the FLIP in predicting clinical outcomes and guiding treatment decisions is exceptionally documented in the adult population for a variety of conditions. Findings related to the 
FLIP described in adult papers do not, however, translate directly into pediatrics considering the drastic differences between children and adults as well as the difference in ways growth and development may present. The potential utility of the FLIP in pediatric is further unknown because there is currently no standardized protocol for its use in children, placing the onus onto the provider to enact their own protocol.

In this review, we seek to delineate currently existing literature describing the use of the EndoFLIP and EsoFLIP in the pediatric population and to describe our experience with the technology thus far.

\section{Review of Current Literature}

\section{EndoFLIP 1.0 and 2.0 Systems}

As previously described, the EndoFLIP uses impedance planimetry to decipher pressure and CSA relationships during volumetric distensions, in turn providing distensibility index and compliance [2]. The first iteration of the EndoFLIP system, EndoFLIP 1.0, first received FDA approval in 2009 and provides real-time measurements of esophageal morphology within esophagus and sphincters of the alimentary canal $[3,4]$.

The most recent iteration of the EndoFLIP system, EndoFLIP 2.0, first received FDA approval in 2017 and is a more sophisticated, advanced departure from the prior system [5]. In addition to a larger screen, EndoFLIP 2.0 is able to measure contractility patterns, screen for motility disorders in real time, and most notably features the Flip Topography module [4].

The EsoFLIP catheter is an interface to the EndoFLIP system which received FDA approval in 2014. The EsoFLIP catheter is attached to either the EndoFLIP 1.0 or 2.0 units and is used for balloon dilation procedures [6,7]. EsoFLIP yields a comprehensive view of the esophagus while dilating, allowing for the accurate measure of stricture size prior to and following each stage of balloon dilation [8].

\section{Achalasia}

The utility of the EndoFLIP in both the pediatric and adult populations is perhaps best documented as an adjunct to peroral endoscopic myotomy (POEM) and laparoscopic Heller myotomy (LHM) procedures in achalasia patients. Adult studies describe the use of the EndoFLIP in providing real-time distensibility measurements of the esophogastric junction (EGJ) and in assessing immediate treatment effects after POEM in large populations. Further, adult studies indicate greater utility in POEM and LHM procedures, with many studies establishing DI and CSA criteria for predicting treatment and symptom outcomes following procedure $[9,10]$. Teitelbaum et al found that intraoperative EGJ distensibility measurements were predictive of postoperative symptomatic outcomes, with an ideal postoperative DI range of $4.5-8.5 \mathrm{~mm}^{2} / \mathrm{mmHg}$ having a sensitivity of $68 \%$ and a specificity of $80 \%$ in predicting optimal symptomatic results and a much greater improvement in DI seen in POEM rather than LHM9. Another multicenter study in adult achalasia patients found that those with lower intraoperative postoperative EGJ CSA were more likely to have persistent symptoms following POEM while higher postoperative EGJ CSA was associated with post-procedure reflux [10]. Although predictive value of EndoFLIP measurements described in the aforementioned publications would certainly be of significant utility in the pediatric achalasia population undergoing POEM, these values have yet to be described in pediatric literature, necessitating larger scale prospective studies with long-term follow up.

While findings from adult papers are informative, they cannot be applied directly to pediatrics due to anatomical and physiological differences between adults and children. Two pediatric papers have echoed findings in adults, detailing the utility of the EndoFLIP in allowing for real-time distensibility measurements of the EGJ as well as assessing of immediate treatment effects after POEM. In a case series of twenty-one pediatric achalasia patients who underwent POEM, researchers found that the nineteen patients who had EndoFLIP measurements had a median preoperative distensibility index (DI) of $1.0 \mathrm{~mm}^{2} / \mathrm{mmHg}$, which improved to $3.5 \mathrm{~mm} 2 / \mathrm{mmHg}$ $(S D=0.4, p<0.0001)$ postoperatively [11]. Another case study which described the first case of pediatric achalasia managed with POEM incorporated EndoFLIP and double endoscope detailed similar results, with a preoperative DI of $1.7 \mathrm{~mm} 2 / \mathrm{mmHg}$ and a markedly increased postoperative DI of $6.0 \mathrm{~mm}^{2} / \mathrm{mmHg}$ [12].

\section{Esophageal Stenosis}

Most recently, a retrospective study involving a total of 19 FLIP procedures in 18 patients at a single pediatric center described the utility of both EsoFLIP and EndoFLIP in patients with esophageal stenosis [13]. Results suggested that dilation using EsoFLIP may yield larger diameter change and reduce procedural time compared to traditional balloon dilation, as patients undergoing EndoFLIP with traditional balloon dilation had a median procedure time of 60.5 minutes and those undergoing only EsoFLIP had a median procedure time of 35 minutes. While insignificant, the patient group who underwent EndoFLIP plus traditional balloon dilation was associated with a smaller diameter increase compared to the group who underwent EsoFLIP ( $\mathrm{p}=0.09$ ). The authors additionally suggested that knowledge of crucial luminal parameters, such as compliance and distensibility, may guide endoscopic interventions more efficiently by helping the endoscopist to determine the amount of dilation necessary and quantify how much the esophagus has been dilated which in turn bears the potential for a reduction in scar tissue formation post-intervention. EsoFLIP evidently is a reasonable alternative to traditional balloon dilation in pediatric patients, however further well-powered studies comparing the EsoFLIP against traditional dilation tools should be performed to provide greater generalizability. 
The use of the EsoFLIP in pediatric patients with esophageal stenosis was first described in a case report in which the EsoFLIP was used to dilate a tracheoesophageal fistula-related stricture [14]. The patient tolerated the procedure well and showed complete resolution of their dysphagia and food intolerance three months later. As fluoroscopy was not required, the authors suggested that EsoFLIP has potential to significantly reduce radiation exposure in pediatric patients, specifically in those who require multiple dilations to resolve strictures.

\section{Eosinophilic Esophagitis (EoE)}

Based on current literature, we are best able to appreciate the utility of the EndoFLIP in the pediatric population in patients with eosinophilic esophagitis (EoE). The primary management outcome in EoE is histology, however it is not without limitations as EoE is a patchy disease and esophageal eosinophilia may not correlate with symptoms and may be resultant of another cause [15]. The utility of the EndoFLIP in the management of EoE is of particular interest as it bears the potential of acting as its first standardized functional parameter. The influence of EoE on esophageal distensibility in pediatric patients was first described in a prospective observational study at two pediatric centers in a cohort of $88 \mathrm{EoE}$ and 44 non-EoE control patients who had normal upper endoscopy with normal esophageal and gastric biopsies [16]. This study demonstrated for the first time that pediatric esophageal distensibility, determined by minimal esophageal body diameter at maximal intrabag pressure, is significantly decreased in children with active EoE compared to non-EoE controls $(\mathrm{p}<0.0001)$ and that distensibility increased with age in non-EoE controls $(\mathrm{p}=0.0001)$. The study additionally described that EoE subjects with significantly active inflammation (eos> 15 eos /hpf) had decreased distensibility compared to EoE subjects with inactive inflammation ( $p=0.0007)$, suggesting that an ability to reverse or even stop fibrotic remodeling when inflammation is treated in children may exist, further highlighting the importance of early and ongoing therapy.

A more recent study describes the utility of esophageal compliance in quantifying epithelial remodeling using a cohort of 11 EoE patients and 12 non-EoE control patients [2]. Contrary to the prior study, it did not yield the same significance in terms of distensibility but did find that esophageal compliance was significantly lower in EoE patients compared to non-EoE controls. While this publication was one of the first to describe the utility of the EndoFLIP in the pediatric population as well as in those with EoE, it is limited by a small patient population and by a less refined protocol given our limited experience.

\section{Site Experiences}

Children's Hospital of Philadelphia (CHOP) pioneered the use of FLIP as a research tool in a pediatric setting starting in 2014. In collaboration with Children's Hospital of Colorado, CHOP's team evaluated esophageal distensibility in pediatric patients with
EoE and non-EoE controls using the EndoFLIP 1.0 unit [16]. An adaptation of the manufacturer's protocol was used to measure esophageal distensibility (Crospon, Galway) [17]. The protocol used in pediatric EoE and non-EoE controls was described by MenardCatcher et al. [16]. FLIP catheters where used based on the height of the patients, EF325N in children $<48$ inches tall and EF322N in in children $>48$ inches in height. Children undergoing sedated EGD underwent FLIP procedure. The FLIP catheter was inserted alongside the endoscope until the tip was positioned beyond the EGJ. The balloon was then inflated to $20 \mathrm{~mL}$ and the catheter repositioned, keeping two leads below the EGJ, as visualized in the unit's display. The FLIP balloon was then inflated in $10 \mathrm{~mL}$ increments with 5 to 20 second holds to a maximum volume of 65 $\mathrm{mL}$ with the EF-322 catheter and $50 \mathrm{~mL}$ with the EF-325 catheter. In the instance of an increase in pressure $>50 \mathrm{mmHg}$, the balloon was immediately deflated. After completion of the FLIP procedure the FLIP balloon was deflated to $0 \mathrm{~mL}$ then withdrawn. Data was transferred to a flash drive and stored for further processing. Data was filtered to eliminate the effect of respirations using a STATA code. Esophageal distensibility was defined as the minimal esophageal body diameter at the point of maximal esophageal distension at an intrabag pressure of $40 \mathrm{mmHg}$.

The clinical use of FLIP at CHOP was expanded after its pediatric indication in 2019, making its use available to children 5 years of age and older [18]. Mainly, FLIP is used at CHOP to evaluate esophageal morphology and function in patients reporting upper esophageal symptoms (i.e. dysphagia, regurgitation, food impactions). The greatest clinical utility of FLIP at CHOP has been in patients with achalasia undergoing balloon dilations. There is a significant amount of literature describing the use of FLIP in adult patients with achalasia that supports its potential benefit in pediatrics $[19,20]$. At CHOP, FLIP is used to measure EGJ distensibility prior to an immediately after a balloon dilatation in patients with achalasia using the EF-325N catheter. We can observe an immediate increase in EGJ-DI immediately after balloon dilatations in the majority of patients. Our team has had a positive experience with FLIP in this population and seen the potential to improve care through its use. Additional uses of FLIP at CHOP include patients with known esophageal strictures, more commonly those with TEF repair. In these cases, localization of the narrowed segment is key for appropriate therapeutic intervention. Since then, esophageal FLIP protocols in pediatrics have evolved with longer holds during stepwise distension of the FLIP balloon and changes in maximum volume of inflation. Finally, we have recently applied the use of FLIP to measure pyloric distensibility in patients with gastroparesis and to rule out pyloric strictures. However, the lack of standardized pediatric protocols limits the expansion and utility of this technology in children.

At Rady Children's Hospital, we have been using the EndoFLIP for almost five years and have performed nearly 100 EndoFLIP 
procedures, all of which were performed as a routine assessment for esophageal dysphagia and occurred during clinically indicated esophagogastroduodenoscopy (EGD) procedures. Our patients had conditions inclusive of eosinophilic esophagitis (EoE), achalasia, TEF, esophageal dysphagia, functional dysphagia, supragastric belching, GERD, or gastritis, although the majority of the patients who have had the EndoFLIP procedure at our center had eosinophilic esophagitis. In collaboration with our colleagues from biomedical engineering, we used same acquisition protocol and created our own automated analysis protocol. Acquisition protocol includes positioning the catheter balloon in two different locations: first at the gastroesophageal junction and in the esophageal body (defined as the mid-point between the upper and lower esophageal junctions). At each placement, the catheter was inflated in serial volumetric distensions with first distension to $20 \mathrm{~mL}$, then by $5 \mathrm{~mL}$ increment to a maximum of $40 \mathrm{~mL}$ (325 N) or $60 \mathrm{~mL}$ (322 N). Each volume was maintained for 30 seconds. Distensibility was assessed by pressure readings at each volume, with the procedure being terminated once a pressure of $60 \mathrm{mmHg}$ or maximum volume was reached. For all EndoFLIP procedures, esophageal compliance was calculated at the proximal, mid, and distal esophageal body as well as the lower esophageal sphincter using the pressure to volume curve generated at each placement. Compliance measurement at the LES was added to our protocol in August 2018 yielding two compliance curves for each procedure, one at the LES and one for the esophageal body.

\section{Future Directions/Conclusion}

We believe that the FLIP has great potential to be of routine clinical use in pediatrics both as an adjunct to procedures and even on its own based off of the current literature in conjunction with our own experience. Across all studies, the safety of the EndoFLIP in the pediatric population has been consistently documented, providing an additional reason to promote its use. Although great potential is on the horizon for the use of FLIP, literature describing its use in pediatrics is few and far between. Pediatric studies are further limited as there are little to no studies describing EndoFLIP measurements in normal children, making it difficult to define a normal baseline to compare patients against. Additionally, pediatric patients in control groups of current EndoFLIP literature may not necessarily be defined as "healthy controls" as all underwent clinically indicated endoscopy. Lastly, current pediatric studies suffer from study populations which are too small or from control groups that are significantly smaller than that of the case group. Lack of studies detailing the use of the FLIP in pediatric POEM and LHM may be due in part to such procedures being done less in children in addition to the decreased prevalence of achalasia in the pediatric population [21].

All in all, we look forward to utilizing the FLIP more and more for both diagnostic and therapeutic reasons and believe that its great utility in pediatrics will boost its relevance in pediatric clinical care. Its utility, however, must overall be better documented in the literature more frequently and in the setting of a controlled prospective study in a large, diverse population.

\section{Acknowledgements}

\section{Conflict of Interest}

The authors declare that they have no conflict of interest.

\section{Reference}

1. Su B, Callahan ZM, Novak S, Kuchta K, Ujiki MB (2020) Using Impedance Planimetry (EndoFLIP) to Evaluate Myotomy and Predict Outcomes After Surgery for Achalasia. J Gastrointest Surg 24(4): 964-971.

2. Hassan M, Aceves S, Dohil R, Armen Gharibans, Robert Newbury, et al. (2019) Esophageal Compliance Quantifies Epithelial Remodeling in Pediatric Patients with Eosinophilic Esophagitis. J Pediatr Gastroenterol Nutr 68(4):559-565.

3. Morris JM (2009) EndoFLIP 510k Clearance K092850.

4. (2017) Flip Technology.

5. Fisher BR (2017) FLIP Topography 510(k) Clearance K170833.

6. Fisher BR (2014) EsoFLIP ES-330 510(k) Clearance K1322337.

7. Fisher BR (2014) EsoFLIP ES-320 510(k) Clearance K142000.

8. (2017) Esophageal Treatment.

9. Teitelbaum EN, Soper NJ, Pandolfino JE, Peter J Kahrilas, Ikuo Hirano, et al. (2015) Esophagogastric junction distensibility measurements during Heller myotomy and POEM for achalasia predict postoperative symptomatic outcomes. Surg Endosc 29(3): 522-528.

10. Ngamruengphong S, von Rahden BHA, Filser J, Amy Tyberg, Amit Desai, et al. (2016) Intraoperative measurement of esophagogastric junction cross-sectional area by impedance planimetry correlates with clinical outcomes of peroral endoscopic myotomy for achalasia: a multicenter study. Surg Endosc 30(7): 2886-2894.

11. Wood LS, Chandler JM, Portelli KE, Taylor JS, Kethman WC, et al. (2020) Treating children with achalasia using per-oral endoscopic myotomy (POEM): Twenty-one cases in review. J Pediatr Surg 55(6): 1006-1012.

12. Yeung F, Wong IYH, Chung PHY, Wong KKY, Law SYK, et al. (2017) Peroral Endoscopic Myotomy with EndoFLIP and Double-Endoscope: Novel Techniques for Achalasia in Pediatric Population. J Laparoendosc Adv Surg Tech 28(3): 343-347.

13. Ng K, Mogul D, Hollier J, Khashab MA (2020) Utility of functional lumen imaging probe in esophageal measurements and dilations: a single pediatric center experience. Surg Endosc 34(3): 1294-1299.

14. Taylor JS, Danzer E, Berquist WE, Wall JK. (2018) Dilation of Esophageal Stricture in a Pediatric Patient Using Functional Lumen Imaging Probe Technology Without the Use of Fluoroscopy. J Pediatr Gastroenterol Nutr 67(2): e20-e21.

15. Muir AB, Merves J, Liacouras CA (2016) Role of Endoscopy in Diagnosis and Management of Pediatric Eosinophilic Esophagitis. Gastrointest Endosc Clin N Am 26(1): 187-200.

16. Menard-Katcher C, Benitez AJ, Pan Z, Faria N Ahmed, Benjamin J Wilkins, et al. (2017) Influence of Age and Eosinophilic Esophagitis on Esophageal Distensibility in a Pediatric Cohort. Am J Gastroenterol 112(9): 1466-1473.

17. Kwiatek MA, Hirano I, Kahrilas PJ, Rothe J, Luger D, et al. (2011) Mechanical properties of the esophagus in eosinophilic esophagitis. Gastroenterology 40(1): 82-90. 
18. Fisher BR (2019) EndoFlip 510k Clearance K183072.

19. Donnan EN, Pandolfino JE (2020) Applying the Functional Luminal Imaging Probe to Esophageal Disorders. Curr Gastroenterol Rep 22(3): 10.

20. Chen HM, Li BW, Li LY, Liang Xia, Xiang Bo Chen, et al. (2019) Functional lumen imaging probe in gastrointestinal motility diseases. J Dig Dis 20(11): 572-577.
21. Petrosyan M, Khalafallah AM, Guzzetta PC, Sandler AD, Darbari A, et al. (2016) Surgical management of esophageal achalasia: Evolution of an institutional approach to minimally invasive repair. J Pediatr Surg 51(10): 1619-1622. 